\title{
Estrategias de afrontamiento como variables predictivas de la intensidad sintomatológica y el cambio clínico tras un tratamiento cognitivo-conductual para el trastorno adaptativo
}

\author{
FABIOLA REYES-TORRES \\ freyes@uji.es \\ IRYNA RACHYLA \\ rachyla@uji.es \\ SILVIA FUENTES-CERDA \\ al287715@uji.es \\ MAR MOLÉS AMPOSTA \\ molesm@psb.uji.es \\ SOLEDAD QUERO CASTELLANO \\ squero@uji.es
}

\section{Resumen}

Introducción: Se ha encontrado que en el contexto de afecciones médicas el afrontamiento activo se relaciona con una mejor respuesta al tratamiento. Sin embargo, no se ha analizado su influencia en el tratamiento de los Trastornos Adaptativos (TA). Objetivo: Analizar la capacidad predictiva de los estilos de afrontamiento en la intensidad sintomática y en el cambio clínico en pacientes con TA. Método: La muestra incluyó 44 pacientes que recibieron un TCC de 6-8 sesiones para los TA. El cuestionario Brief COPE (Carver, 1997) se utilizó para medir las estrategias de afrontamiento. La sintomatología depresiva y de estrés y pérdida fue evaluada en el post-tratamiento y seguimientos de los 3, 6 y 12 meses. Resultados: Las estrategias de afrontamiento no predijeron la intensidad sintomatológica depresiva pero sí la asociada al estrés y pérdida. Estos síntomas fueron menos intensos en pacientes que utilizaban en mayor grado afrontamiento cognitivo y en menor las estrategias de bloqueo y evitación. En cuanto al cambio clínico, en el post y los seguimientos de los 3 y 12 meses los resultados del tratamiento eran independientes de los estilos de afrontamiento iniciales. No obstante, el mayor uso de apoyo social predijo una mayor mejoría en estrés y pérdida en el seguimiento de los 6 meses. Conclusiones: Los resultados subrayan el papel predictor del afrontamiento activo (cognitivo y social) en la mejoría de los síntomas relacionados con el acontecimiento estresante. Se sugiere promover el uso de estrategias de afrontamiento adaptativas durante el tratamiento de los TA.

Palabras clave: trastorno adaptativo, estrategias de afrontamiento, cambio clínico, depresión, estrés y pérdida. 


\section{Abstract}

Introduction: It has been found that in the field of medical afflictions active coping is related with a better response to treatment. However, the influence of coping strategies on Adjustment Disorder (AD) has not been sufficiently analyzed. Aim: To explore the predictive value of coping styles to predict the severity of symptoms and clinical change in patients with AD. Method: The sample included 44 patients who received a 6-8 session CBT for AD. Brief COPE questionnaire (Carver, 1997) was used to measure coping strategies. Depressive and stress and loss symptoms were assessed at post-intervention and 3, 6 and 12 months follow-ups. Results: Coping strategies did not predict the severity of depressive symptoms, however, they showed significant relationship with the severity of symptoms related to stress and loss. These symptoms were less severe in patients who used more cognitive coping strategies and less blocking or avoidance strategies. Regarding to clinical change, treatment outcomes were independent of the initial coping styles at post-intervention and 3 and 12 months follow-up. Nevertheless, greater use of social support predicted a more significant improvement in stress and loss symptoms at 6 months follow-up. Conclusions: Results highlight the predictive role of active coping (cognitive and social) in the improvement of symptoms related to the stressful event. It is recommended to promote the use of adaptive coping strategies during the treatment of $A D$.

Keywords: adjusment disorder, coping strategies, meaningful change, depression, stress and loss.

\section{Introducción}

Las estrategias de afrontamiento constituyen el conjunto de acciones que un individuo despliega ante situaciones altamente amenazantes con el propósito de detener, atenuar o bien anular sus efectos emocionales facilitando así la adaptación a las mismas (Morán, Landero \& González, 2010). De esta manera, estrategias de afrontamiento adaptativas conducen a un mejor manejo del estrés y a un mayor ajuste a la situación (Lazarus, Delongis, Folkman \& Gruen, 1985). La adopción de un determinado tipo de estrategia dependerá de la forma en que se valore el suceso estresante. Existen dos tipos de afrontamiento: cuando la situación es evaluada como fácil de resolver normalmente se utilizan estrategias centradas en la resolución del problema, por el contrario, cuando la situación es percibida como dañina o desafiante y, por tanto, difícil de cambiar, suelen utilizarse estrategias centradas en la emoción, las cuales se centran específicamente en cambiar las emociones asociadas a la situación (Folkman, 1984).

En el ámbito clínico, la evidencia ha mostrado que las estrategias de afrontamiento determinan la intensidad sintomatológica asociada a diferentes trastornos emocionales. En relación al trastorno depresivo, Burns \& Nolen-Hoeksem (1991) demostraron que las personas que empleaban con menor frecuencia estrategias de afrontamiento positivo presentaban mayor severidad depresiva, en comparación con aquellas que las utilizaban de forma habitual. En un estudio reciente se encontró que la gravedad sintomatológica de los síndromes subclínicos de depresión, trastorno obsesivo compulsivo y paranoia correlacionaba fuertemente con 
el afrontamiento desadaptativo, no obstante, la magnitud de la relación era mucho más fuerte para los síntomas depresivos (Moritz et al., 2016). Estos resultados se confirman con los hallados en el estudio de Potthoff et al. (2016), donde estrategias desadaptativas como la autoinculpación y la catastrofización fueron capaces de predecir altos niveles de depresión.

En cuanto a la relación entre los estilos de afrontamiento y la sintomatología ansiosa; se han encontrado asociaciones fuertes entre la catastrofización, la rumiación, la auto-inculpación y la intensidad de las puntuaciones de ansiedad informadas (Potthoff et al., 2016). Asimismo, una investigación llevada a cabo en pacientes con trastorno de pánico, arrojó que éstos usaban con menor frecuencia estrategias de afrontamiento efectivas (solución de problemas y reevaluación positiva), en comparación con el grupo control (Savoia \& Bernik, 2004).

Los hallazgos anteriores revelan el carácter predictivo de las estrategias de afrontamiento en la gravedad sintomática de trastornos como la depresión y la ansiedad, no obstante, se ha dedicado escasa atención a su influencia en otro tipo de desórdenes como el Trastorno Adaptativo (TA). De acuerdo a los criterios propuestos por el DSM-5 (APA, 2013), el TA puede definirse como una serie de reacciones negativas, tanto en el ámbito emocional como conductal, que se despliegan ante la experiencia de un evento vital estresante o ante la adversidad; generalmente se presenta dentro de los 3 meses posteriores a la ocurrencia del suceso. Uno de los aspectos fundamentales para el diagnóstico es que el malestar emocional debe ser desproporcionado en relación a la intensidad del factor estresante, tomando en cuenta siempre el contexto externo y los aspectos culturales. Además, el malestar debe interferir de forma significativa en el funcionamiento social, laboral, escolar o en las relaciones interpersonales.

Como se ha mencionado, el papel del afrontamiento en el TA no ha sido suficientemente investigado. No obstante, dado que las enfermedades físicas constituyen en la mayoría de los casos un cambio vital acompañado de grandes cargas de estrés, uno de los campos que ha aportado resultados importantes en esta área es el de las afecciones médicas. Por ejemplo, en pacientes con enfermedades cardíacas, el mayor uso de estrategias de afrontamiento desadaptativas se ha relacionado con síntomas depresivos más intensos, en contraste, se ha encontrado que personas con problemas cardíacos crónicos que adoptan estrategias de afrontamiento adaptativas se encuentran menos expuestas a desarrollar depresión (Holahan, Moos, Holahan y Brennan, 1995; Pérez-García, Oliván \& Bover, 2013). En la misma línea, Giammanco \& Gitto (2016) evidenciaron en pacientes cardíacos hospitalizados que el uso de estrategias activas (reinterpretación positiva y crecimiento) correlacionaba negativamente con síntomas de ansiedad y depresión tanto extremos como patológicos. También se observó que las estrategias pasivas de afrontamiento se relacionaban positivamente con puntuaciones altas de ansiedad.

Por otro lado, la investigación empírica también ha encontrado que el afrontamiento guarda relación con la mejoría clínica. Así, Hall, Marshall, Mercado \& Tkachuk (2011) midieron el cambio clínico en personas que habían sufrido un accidente de tráfico después de ser sometidas a tratamiento y sus resultados revelaron que, tras la intervención, aquellos pacientes que adoptaban una afrontamiento activo hacia la rehabilitación (evitando el afrontamiento pasivo), informaron mayores beneficios tanto en las puntuaciones de calidad de vida como en su funcionalidad física, en comparación con aquellos que no lo hicieron.

En el ámbito de los trastornos de ansiedad las estrategias de afrontamiento han mostrado un comportamiento similar. En un estudio realizado con pacientes con trastorno de pánico que habían recibido una intervención cognitivo-conductual grupal, se observó posterior a la terapia un uso menos frecuente de estrategias como la confrontación, el escape y la evitación; además como resultado del tratamiento también incrementó el uso de estrategias adaptativas, aspecto que se relacionó con una disminución de la frecuencia de los ataques de pánico y de las puntuaciones de ansiedad anticipatoria (Wesner et al., 2014). Investigaciones recientes 
también han revelado que el afrontamiento cognitivo funciona como un mecanismo de cambio en pacientes con miedo a volar pues después de un tratamiento cognitivo-conductual se observó una reducción significativa en el uso del afrontamiento desadaptativo (auto-inculpación, rumiación, catastrofización y evitación) y un incremento significativo en las estrategias adaptativas (reenfoque positivo y reevaluación positiva). Además, ambos resultados fueron predictores de mejoras en el miedo a volar auto-informado tanto a corto como a mediano plazo (Busscher \& Spinhoven, 2016).

Por último, en lo referido a la investigación sobre el TA, se ha encontrado que contar con características personales como la extroversión, tener vínculos familiares fuertes y recibir apoyo y consuelo de parte de los miembros de la red social (amigos), constituyen factores que previenen el desarrollo de trastornos emocionales inducidos por estrés; de forma inversa el uso inapropiado de las estrategias de afrontamiento y de los recursos sociales incrementa el riesgo de su aparación (Kornblith et al., 2001). Finalmente, el estudio llevado a cabo por Ponizovsky, Levov, Schultz \& Radomislensky (2011), reveló que los participantes con TA tenían más probabilidad de usar un patrón de afrontamiento orientado hacia las emociones ante situaciones estresantes, mientras que la población no clínica tendía a utilizar estrategias centradas en tareas; también encontraron que los participantes con TA usaban significativamente menos el afrontamiento mediante búsqueda de apoyo social y tenían una percepción disminuída de la ayuda recibida de parte de familiares y pares. Un hallazago relevante derivado de este estudio fue la asociación predictiva encontrada entre un deficiente apoyo social y la intensidad de los síntomas depresivos.

Tomando como base la evidencia empírica que confirma que el afrontamiento constituye una variable tanto predictiva como de cambio clínico en un amplio espectro de trastornos y, atendiendo a la escasa investigación que existe en el campo de los TA, el presente estudio plantea la necesidad de explorar con mayor profundidad la manera en que el afrontamiento tanto adaptativo como desadaptativo opera en las variables clínicas asociadas a este trastorno. Así, el objetivo de este estudio es conocer la capacidad predicitiva de las estrategias de afrontamiento en la intensidad sintomatológica pre-tratamiento, por un lado, y en el cambio clínico en pacientes diagnosticados con TA tras recibir una intervención cognitivo conductual, por otro.

\section{Método}

\section{Participantes}

La muestra del estudio estuvo compuesta por 44 pacientes (33 mujeres y 11 hombres) con diagnóstico de TA (APA, 2013), cuya media de edad fue de 29,09 años (DT = 8,95). El 75 $\%$ de participantes contaba con estudios universitarios y el $25 \%$ restante tenían formación básica (primaria y/o secundaria). En cuanto a la distribución de los subtipos clínicos, el 70,5 $\%$ presentaban TA adaptativo mixto (con síntomas depresivos y ansiosos), el 15,9\% cumplían los criterios para el diagnóstico de TA con estado de ánimo depresivo, el 11,4 \% fue diagnosticado con TA con ansiedad y el 2,3\% presentaba alteración mixta de emociones y comportamiento. 


\section{Medidas}

Medidas para las estrategias de afrontamiento

Escala Multidimensional de Estilos de Afrontamiento (Abreviado) (Brief COPE; Carver, 1997). Es un instrumento que evalúa las estrategias de afrontamiento, tanto efectivas como ineficaces. Consta de 28 preguntas que se responden en una escala Likert de 4 alternativas que van de 0 (»no lo hago nunca») a 3 (»lo hago con mucha frecuencia»). Se compone de 14 sub-escalas (de 2 ítems cada una) que pueden agruparse bajo 4 factores (Morán, Landero \& Gozález, 2010): 1) Afrontamiento cognitivo, que incluye las sub-escalas de afrontamiento activo, planificación, reinterpretación positiva, humor y desconexión (inverso); 2) Afrontamiento mediante apoyo social, que incluye las sub-escalas de apoyo (emocional y social) y desahogo; 3) Bloqueo del afrontamiento (evitación), que agrupa las sub-escalas de negación, auto-distracción, auto-inculpación y uso de sustancias; y 4) Afrontamiento espiritual compuesto únicamente por la sub-escala de religión. La versión española ha demostrado una buena consistencia interna ( $\alpha$ de Cronbach entre 0,60 y 0,90) (Crespo \& Cruzado, 1997).

Medidas para determinar la intensidad sintomatológica

Inventario de Depresión de Beck-II (BDI-II; Beck, Steer \& Brown, 1996). Es uno de los instrumentos más usados para medir la severidad de los síntomas y cogniciones relacionadas con la depresión. Consta de 21 ítems que se puntúan de 0 a 3, ofreciendo una evaluación sencilla del estado de ánimo deprimido. Es sensible a los cambios en los niveles de depresión, por lo que permite determinar la magnitud del cambio clínico. La versión española del instrumento presenta altos niveles de consistencia interna, tanto para la población general ( $\alpha$ de Cronbach de 0,87) (Sanz, Navarro \& Vázquez, 2003) como clínica ( $\alpha$ de Cronbach de 0,89) (Sanz, García-Vera, Espinosa, Fortún \& Vázquez, 2005).

Inventario de Estrés y Pérdida (IEP; Quero, Molés, Mor, Baños \& Botella, 2014). Este instrumento de 17 ítems permite valorar la magnitud tanto de la afectación sintomática como de las alteraciones funcionales que aparecen como consecuencia de vivir una situación estresante. Se trata de una adaptación del Inventario de Duelo Complicado (Prigerson et al., 1995), en la que las expresiones referidas al fallecido fueron sustituidas por otras que aluden a la persona o situación relacionadas con el evento estresante experimentado (p.ej., la pérdida de un trabajo, la pérdida de una pareja, la pérdida de la salud, etc.). El análisis factorial llevado a cabo para el IEP demostró la existencia de un factor único y los resultados preliminares indican que cuenta con un buen coeficiente de consistencia interna en población general y clínica (0,92 y 0,79 , respectivamente), así como una excelentes fiabilidad test-retest $(0,90)$ (Mor, Molés, Rachyla \& Quero, 2015).

\section{Protocolo de intervención}

Todos los participantes recibieron un protocolo de intervención cognitivo-conductual (TCC) para TA (Botella, Baños \& Guillén, 2008), cuyo principal objetivo era reconstruir el significado de las dificultades y los eventos estresantes y aprender de la experiencia vivida. El tratamiento se componía de 6 sesiones (que podían ser ampliadas a 8 en función de las necesidades individuales de cada paciente) de frecuencia semanal y de 90 minutos de duración. En cuanto a los componentes terapéuticos de la intervención, fueron los siguientes: 1) psico- 
educación sobre las reacciones más comunes que se producen en respuesta a un evento estresante, así como sobre el origen y mantenimiento de los TA.; 2) exposición en vivo para superar el malestar vinculado a las situaciones, actividades, lugares o personas relacionadas con el acontecimiento estresante; 3) elaboración del evento estresante que consistía en la evocación y confrontación de las emociones negativas vinculadas a la situación problema con el objetivo de procesar el malestar y encontrarle un significado alternativo más positivo a la situación; 4) prevención de recaídas. Además, se incluyeron estrategias basadas en psicología positiva con el propósito de potenciar la capacidad de resistencia y crecimiento ante la adversidad. Como principal novedad de la intervención, cabe resaltar que se utilizó un sistema flexible de Realidad Virtual (El Mundo de EMMA) durante el procesamiento y elaboración del acontecimiento estresante. Dicho sistema permitió evocar significados y reacciones emocionales asociadas al evento estresante mediante la combinación de distintos elementos simbólicos dentro de un entorno 3D. Una explicación más amplia del protocolo de intervención y del entorno EMMA puede encontrarse en Quero et al. (2017).

\section{Procedimiento}

Todos los participantes fueron voluntarios y firmaron el consentimiento informado para participar en el estudio. La sintomatología clínica se evaluó en el pre y post-tratamiento, así como en seguimientos a los 3, 6 y 12 meses. En cuanto a las estrategias de afrontamiento, éstas sólo se evaluaron entes de la intervención.

\section{Resultados}

En las Tablas 1 y 2 se presentan respectivamente los estadísticos descriptivos de las diferentes escalas de Brief-COPE y de las puntuaciones totales del BDI-II e IEP obtenidas en cada uno de los momentos de evaluación.

Tabla 1

Medias y desviaciones típicas de las 4 escalas de Brief-COPE obtenidas en el pre-tratamiento

\begin{tabular}{lcc}
\hline Estrategias de afrontamiento & \multicolumn{2}{c}{ Pre-trat } \\
\hline Escala de afrontamiento cognitivo & M & DT \\
Escala de afrontamiento mediante apoyo social & 22,20 & 3,50 \\
Escala de bloqueo & 15,95 & 4,09 \\
Escala espiritual & 16,77 & 3,26 \\
\hline
\end{tabular}


Tabla 2

Medias y desviaciones típicas del BDI-II e IEP obtenidas en el pre, post y seguimientos de los 3, 6 y 12 meses

\begin{tabular}{ccccccccccc}
\hline & \multicolumn{2}{c}{ Pre-trat } & \multicolumn{2}{c}{ Post-trat } & \multicolumn{2}{c}{ Seg3m } & \multicolumn{2}{c}{ Seg6m } & \multicolumn{2}{c}{ Seg12m } \\
\cline { 2 - 12 } & M & DT & M & DT & M & DT & M & DT & M & DT \\
\hline BDI-II & 23,20 & 9,59 & 6,18 & 5,55 & 6,00 & 5,03 & 3,33 & 3,72 & 4,56 & 6,72 \\
IEP & 36,15 & 11,14 & 15,31 & 9,87 & 12,81 & 9,96 & 11,11 & 7,91 & 6,11 & 6,25 \\
\hline
\end{tabular}

Con el objetivo de evaluar si las estrategias de afrontamiento influían en la intensidad de la sintomatología clínica, se llevó a cabo un análisis de regresión lineal por pasos. Las cuatro escalas de Brief-COPE fueron incluidas como variables independientes (VI) y las puntuaciones del BDI-II e IEP obtenidas en el pre-tratamiento como variables dependientes (VD). Los resultados del análisis revelaron que ninguna de las escalas de Brief-COPE predijo la intensidad de la sintomatología depresiva en pacientes con TA. Sin embargo, en lo relacionado a los síntomas de estrés y pérdida, el análisis identificó las escalas de afrontamiento cognitivo activo y de bloqueo como variables predictoras de la gravedad sintomática $\left(\mathrm{R}^{2}=0,027 ; t=-2,57\right.$; $\rho<, 014$ y $t=3,32 ; \rho<, 001$ respectivamente).

Con el propósito de explorar en qué medida las estrategias de afrontamiento predecían el cambio clínico en los pacientes con TA, se volvió a realizar el análisis de regresión por pasos, utilizando esta vez como variables dependientes los índices de cambio fiable (ICF) de Jacobson y Truax (1991) calculados para el BDI-II y el IEP en cada uno de los momentos de evaluación (ver Tabla 3).

Tabla 3

Cambio clínicamente significativo observado en el BDI-Il y el IEP en el post-tratamiento y seguimientos a 3, 6 y 12 meses

\begin{tabular}{rrrrr}
\hline & \multicolumn{1}{c}{ Post } & Seg3m & Seg6m & Seg12m \\
\hline BDI-II & & & & \\
Recuperados & $61,4 \%$ & $62,5 \%$ & $85,2 \%$ & $77,8 \%$ \\
Mejorados & $2,3 \%$ & $0 \%$ & $0 \%$ & $0 \%$ \\
No cambio & $36,4 \%$ & $37,5 \%$ & $14,8 \%$ & $22,2 \%$ \\
Deteriorados & $0 \%$ & $0 \%$ & $0 \%$ & $0 \%$ \\
\hline IEP & & & & \\
Recuperados & $71,4 \%$ & $81,3 \%$ & $78,6 \%$ & $0 \%$ \\
Mejorados & $2,4 \%$ & $3,1 \%$ & $0 \%$ & $11,1 \%$ \\
No cambio & $21,4 \%$ & $6,3 \%$ & $17,9 \%$ & $0 \%$ \\
Deteriorados & $4,8 \%$ & $9,4 \%$ & $3,6 \%$ & \\
\hline
\end{tabular}


En cuanto a la capacidad predictiva de las estrategias de afrontamiento para determinar la magnitud del cambio tras la intervención, en las puntuaciones del BDI-II no se encontró ninguna relación significativa entre los ICF calculados y las 4 escalas del Brief-COPE. Resultados similares fueron hallados para IEP en la evaluación post-tratamiento y en los seguimientos a 3 y 12 meses. No obstante, en el seguimiento de los 6 meses la estrategia de afrontamiento mediante apoyo social resultó ser la única variable predictora del cambio clínico, hallándose una asociación negativa entre este tipo de afrontamiento y la magnitud del cambio en los síntomas de estrés y pérdida $\left(R^{2}=0,38 ; t=-4,20 ; \rho<, 000\right)$.

\section{Discusión y conclusiones}

El objetivo del presente trabajo fue determinar en qué medida diferentes estrategias de afrontamiento predecían tanto la intensidad de los síntomas como la mejoría post-tratamiento en pacientes con TA.

En lo referente a la gravedad clínica, los resultados mostraron la ausencia de relaciones significativas entre la sintomatología depresiva y las estrategias de afrontamiento. Sin embargo, sí que se observó que las estrategias de afrontamiento influían en la intensidad de la sintomatología de estrés y pérdida. Concretamente, se encontró que el uso de estrategias tales como el afrontamiento activo, la reinterpretación positiva, la planificación o el humor, funcionaba como factor protector ante la adversidad, por lo que los pacientes presentaban una sintomatología menos intensa al vivir una situación estresante. El efecto opuesto era observado al utilizar estrategias de afrontamiento caracterizadas por el uso de la auto-distracción, la autoinculpación o el uso de sustancias. Los resultados mostraron que la sintomatología clínica relacionada con el acontecimiento estresante fue más intensa en personas que con mayor frecuencia utilizaban estas estrategias de afrontamiento relacionadas con la evitación y el bloqueo. Estos resultados van en la misma línea que los hallados en la literatura en relación a los trastornos de estado de ánimo y ansiedad y que sugieren que el uso de estrategias de afrontamiento relacionadas con la autoinculpación, la catastrofización o la rumiación generalmente se asocia a mayor intensidad sintomática, mientras que el efecto contrario se observa al utilizar estrategias tales como la reinterpretación positiva o el crecimiento (Giammanco \& Gitto 2016; Potthoff et al., 2016).

Con respecto al cambio clínico significativo, los resultados mostraron que la mejora tanto de los síntomas depresivos como de los de estrés y pérdida era independiente de las estrategias de afrontamiento iniciales de los pacientes, tanto en las mediciones post-tratamiento como en los seguimientos de los 3 y 12 meses. Únicamente en el seguimiento a medio plazo (6 meses) se encontró que las personas que habían estado afrontando el estrés antes de recibir el TCC mediante la búsqueda de apoyo en su círculo social presentaban una mayor disminución del malestar. Estos resultados sugieren que el programa de tratamiento utilizado en este estudio es igual de eficaz para todos los pacientes, sin importar los recursos de afrontamiento de los que disponen. Estos hallazgos también van en la misma línea que los encontrados en la literatura. Así, Busscher \& Spinhoven (2016) encontraron que las estrategias de afrontamiento de los pacientes con fobia a volar registradas en la línea base no influían en su respuesta al tratamiento.

El papel predictor del uso de apoyo social en la evolución positiva del TA es un hallazgo interesante, sobre todo teniendo en cuenta los resultados encontrados por Ponizovsky, Levov, Schultz \& Radomislensky (2011). Estos autores observaron que los pacientes con TA presentaban dificultades para adoptar estrategias que implicaban buscar apoyo social y manifestaban una valoración deficiente del apoyo emocional que recibían de parte de familiares y pares. 
Sus resultados también arrojaron que un deficiente apoyo social era capaz de predecir mayor intensidad en determinados síntomas del trastorno. Los resultados conjuntos de ambos trabajos ponen de manifiesto que el entrenamiento en el uso y la explotación del apoyo social puede ser de crucial interés para la prevención y el tratamiento de los TA.

Algunas limitaciones de este trabajo deben tenerse en cuenta. En primer lugar, el tamaño de la muestra. En segundo lugar, no se debe olvidar que las estrategias de afrontamiento únicamente se midieron en la fase de pre-tratamiento, por lo que los resultados no aportan información en cuanto a si hubo o no cambio en el tipo de estrategias de afrontamiento utilizado después de recibir el TCC. Esta sin duda podría ser una línea de investigación futura.

En conclusión, los resultados encontrados confirman el carácter predictivo de las estrategias de afrontamiento en la clínica del TA, por lo que sería relevante promover el conocimiento y uso de estrategias adecuadas de afrontamiento a nivel preventivo. Por tanto, proponemos la creación de programas de prevención dirigidos a población no clínica que incluyan dichas estrategias con el propósito de reducir la vulnerabilidad al desarrollo de trastornos emocionales inducidos por el estrés.

\section{Referencias Bibliográficas}

APA. Asociación Americana de Psiquiatría (2013). Manual Diagnóstico y Estadístico de los Trastornos Mentales DSM-5 (5a Ed). Washington: American Psychiatric Association.

Beck, A. T., Steer, R. A. \& Brown, G. (1996). Manual for the Beck Depression Inventory-II. San Antonio, TX: Psychological Corp.

Botella, C., Baños, R. M. \& Guillen, V. (2008). Una propuesta de tratamiento para los trastornos adaptatios: creciendo en la adversidad. En C. Vázquez \& G. Hervás (dirs.), Psicología positiva aplicada (pp. 129-154). Bilbao: Desclee de Brouwer.

Burns, D. D. \& Nolen-Hoeksema, S. (1991). Coping styles, homework compliance, and the effectiveness of cognitive-behavioral therapy. Journal of Consulting and Clinical Psychology, 59(2), 305-311. http://doi.org/10.1037/0022-006X.59.2.305

Busscher, B. \& Spinhoven, P. (2016). Cognitive Coping as a Mechanism of Change in Cognitive-Behavioral Therapy for Fear of Flying: A Longitudinal Study With 3-Year Follow-Up. Journal of Clinical Psychology, 0, 1-12. http://doi.org/10.1002/jclp.22424

Carver, C. S. (1997). You want to measure coping but your protocol's too long: Consider the brief cope. International Journal of Behavioral Medicine, 4, 92-100. http://doi.org/10.1207/ s15327558ijbm0401_6

Crespo, M. \& Cruzado, J. A. (1997). La evaluación del afrontamiento: adaptación española del cuestionario COPE con una muestra de estudiantes universitarios. Análisis y Modificación de Conducta, 23, 797-830.

Folkman, S. (1984). Personal control and stress and coping processes: a theoretical analysis. Journal of Personality and Social Psychology, 46, 839-52. Retrieved from http://www. ncbi.nlm.nih.gov/pubmed/6737195

Giammanco, M. D. \& Gitto, L. (2016). Coping, uncertainty and health-related quality of life as determinants of anxiety and depression on a sample of hospitalized cardiac patients in Southern Italy. Quality of Life Research, 25, 2941-2956. http://doi.org/10.1007/s11136016-1323-5

Hall, P. A, Marshall, J., Mercado, A. \& Tkachuk, G. (2011). Changes in coping style and treatment outcome following motor vehicle accident. Rehabilitation Psychology, 56, 43-51. http://doi.org/10.1037/a0022443 
Holahan, C. J., Moos, R. H., Holahan, C. K. \& Brennan, P. L. (1995). Social support, coping, and depressive symptoms in a late-middle-aged sample of patients reporting cardiac illness. Health Psychology, 14, 152-163. http://doi.org/10.1037/0278-6133.14.2.152

Jacobson, N. S. \& Truax, P. (1991). Clinical significance: A statistical approach to defining meaningful change in psychotherapy research. Journal of Consulting and Clinical Psychology, 59, 12-19.

Kornblith, A. B., Herndon, J. E., Zuckerman, E., Viscoli, C. M., Horwitz, R. I., Cooper, M. R., ... Holland, J. C. (2001). Social support as a buffer to the psychological impact of stressful life events in women with breast cancer. Cancer, 91, 443-454. http://doi.org/10.1002/10970142(20010115)91:2<443::AID-CNCR1020>3.0.CO;2-Z

Lazarus, R. S., Delongis, A., Folkman, S. \& Gruen, R. (1985). Stress and Adaptional Outcomes, The Problem of Confounded Measures. American Psychologist, (7), 770-779. http://doi.org/10.1037/0003-066X.40.7.770

Mor, S., Molés, M., Rachyla, I. \& Quero, S. (2015). Validación española del inventario de estrés y pérdida adaptado del inventario de duelo complicado para los trastornos adaptativos. Póster presentado en las I Jornadas de Recerca per als estudiants de Psicologia de la Facultat de Ciències de la Salut. Universitat Jaume I. Castellón.

Morán, C., Landero, R. \& González, M. T. (2010). COPE-28: Un análisis psicométrico de la versión en Español del Brief COPE. Universitas Psychologica, 9, 543-552.

Moritz, S., Jahns, A. K., Schröder, J., Berger, T., Lincoln, T. M., Klein, J. P. \& Göritz, A. S. (2016). More adaptive versus less maladaptive coping: What is more predictive of symptom severity? Development of a new scale to investigate coping profiles across different psychopathological syndromes. Journal of Affective Disorders, 191, 300-307. http://doi. org/10.1016/j.jad.2015.11.027

Pérez-García, A. M., Oliván, S. \& Bover, R. (2013). Subjective Well-being in Heart Failure Patients: Influence of Coping and Depressive Symptoms. http://doi.org/10.1007/s12529-013 $-9311-4$

Ponizovsky, A. M., Levov, K., Schultz, Y. \& Radomislensky, I. (2011). Attachment Insecurity and Psychological Resources Associated With Adjustment Disorders. American Journal of Orthopsychiatry, 81, 265-276. http://doi.org/10.1111/j.1939-0025.2011.01095.x

Potthoff, S., Garnefski, N., Miklósi, M., Ubbiali, A., Domínguez-Sánchez, F. J., Martins, E. C., ... Kraaij, V. (2016). Cognitive emotion regulation and psychopathology across cultures: A comparison between six European countries. Personality and Individual Differences, 98, 218-224. http://doi.org/10.1016/j.paid.2016.04.022

Prigerson, H. G., Maciejewsku, P. K., Reynolds, C. F., Bierhals, A. J., Newsom, J. T., Fasiczka, A., ... Miller, M. (1995). Inventory of Complicated Grief: A scale to measure maladaptive symptoms of loss. Psychiatry Research, 59, 65-79.

Quero, S., Andreu-Mateu, S., Moragrega, I., Baños, R. M., Molés, M., Nebot, S., \& Botella, C. (2017). Un programa cognitivo-conductual que utiliza la realidad virtual para el tratamiento de los trastornos adaptativos: Una serie de casos. Revista Argentina de Clínica Psicológica, 36, 5-18.

Quero, S., Molés, M., Mor, S., Baños, R. \& Botella, C. (2014). Spanish Validation of the Complicated Grief Inventory adapted for Adjustment Disorders. Poster presentado en ABCT's 48th Annual Convention. Philadelphia.

Sanz, J., García-Vera, M-P., Espinosa, R., Fortún, M. \& Vázquez, C. (2005). Adaptación española del inventario para la depresión de Beck- II (BDI-II): Propiedades psicométricas en pacientes con trastornos psicológicos. Clínica Y Salud, 16, 121-142. 
Sanz, J., Navarro, M. E. \& Vázquez, C. (2003). Adaptación española del Inventario para la Depresión de Beck-II (BDI-II): Propiedades psicométricas en estudiantes universitarios. Análisis y Modificación de Conducta, 29, 239-288.

Savoia, M. G. \& Bernik, M. (2004). Adverse life events and coping skills in panic disorder, 59, 337-340. Retrieved from http://www.scielo.br/pdf/rhc/v59n6/a05v59n6.pdf

Wesner, A. C., Gomes, J. B., Detzel, T., Blaya, C., Manfro, G. G. \& Heldt, E. (2014). Effect of cognitive-behavioral group therapy for panic disorder in changing coping strategies. Comprehensive Psychiatry, 55, 87-92. http://doi.org/10.1016/j.comppsych.2013.06.008 